\title{
Preliminary Design Of An Electrodynamic Balance For Single Particle Analysis
}

\author{
C. Bobcowski, J. Costa , S. Shaw , R. Zucal', J. Schmalzel, R. Ordoñez' and J. Chen
}

\begin{abstract}
An electrodynamic chamber $(E D C)$ is a device that controls the electrodynamic field surrounding a single particle. By suspending the particle at a fixed point in space, various measurements can be made to determine thermodynamic properties of the particle. This paper describes the preliminary engineering development of an EDC for combustion research at Rowan University.
\end{abstract}

\section{INTRODUCTION}

The main approaches for thermodynamic studies of particles are based on thermo-gravimetric analysis (TGA) using beam balance, calorimetric analysis, screen heating, drop-tube furnace techniques, shock-tube methods, and fluidized bed reactors [1]. These methods are well developed and provide data over a wide range of temperatures and heating rates. The electrodynamic chamber (EDC), sometimes referred to as an electrodynamic balance (EDB), has been developed to study many properties of a single particle. Once a particle has been fixed in space, precise physical and chemical measurements can be made. Some of the testing advantages of the EDC over other techniques are the ability to study the combustion kinetics of a single particle in controlled conditions, the ability to characterize the particle prior to reaction, the capability to monitor quantities needed for kinetic understanding in real time, and elimination of heat and mass transfer limitations, and particle to particle variation.

The EDC operates by creating an electrodynamic field on a charged particle. A constant ac voltage applied to a horizontal ring electrode that surrounds the particle to restrains the horizontal position of the particle and contributes to the vertical force, while a varying $\mathrm{dc}$ voltage difference between the vertical electrodes controls the vertical position of the particle. When the particle is balanced at the center of the EDC, equation (1) holds [1],

$$
\frac{q}{m}=\frac{g z_{0}}{c V_{D C}^{0}}
$$

where $q / m$ is the particle's charge-to-mass ratio, $g$ is acceleration due to gravity, $z_{0}$ is the particle vertical distance from the null point, $c$ is a chamber constant, and
$V_{D C}^{0}$ is the dc voltage required to levitate and hold the particle at the center of the chamber.

Currently, only two known electrodynamic chambers are used for high temperature studies $[1,2,3,4]$. The overall goal of this project is to design and construct a device that electrically levitates a coal particle. More specifically, the objectives of the research described in this paper are to develop a customized design from research of existing chambers, minimizing the chamber "dead space," and creating a hybrid control system. Initially the chamber needed to be designed for a uniform plastic particle with known physical and chemical properties. This project consists of several phases: (i) Preliminary design studies to develop and prototype key elements, (ii) Prototype development, and (iii) Testing of integrated system with levitation and combustion of a coal particle. This paper describes the results of Phase 1 .

\section{EDC DESIGN}

Fig. 1 shows the block diagram of an EDC system for single particle analysis. The dc (end cap) electrodes control the vertical position of the particle. For our design, we assume an initial potential of \pm 300 volts. The ac ring electrode provides lateral and vertical forces to the particle. This essential component notably causes great safety concerns. The ring electrode ac source provides 2000 volts rms at frequencies ranging from 10 to $60 \mathrm{~Hz}$. The constant ac signal is connected to the ring electrode at a single point, and shares a common reference with the dc supply.

The bandwidth for the EDC design

$$
B=\frac{\sqrt{2 g}}{2 \sqrt{d x}}
$$

is calculated by considering the particle falling from the top to the bottom electrode, where $d x$ is the distance between vertical electrodes and $g$ is the acceleration due to gravity. For our design, $B=20.2 \mathrm{~Hz}$. Assuming an over-sampling, conservative approach yields a minimum sampling frequency $\left(f_{s}\right)_{\min } \approx 200 \mathrm{~Hz}$. 


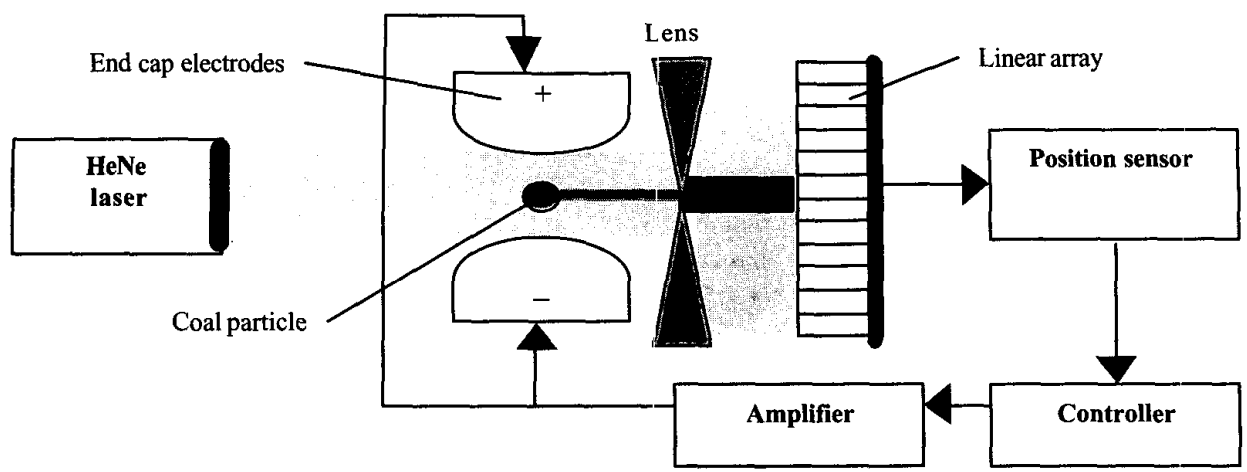

Fig.1. Control system block diagram for EDC (ring electrode not shown).

\section{A. Electrode Geometry}

Two vertical electrodes and a horizontal ring electrode, as shown in Fig. 2, are used to regulate the position of the particle. $r$ is the radial axis, $z$ is cylindrical axis, and the null point is defined as the center of the chamber.

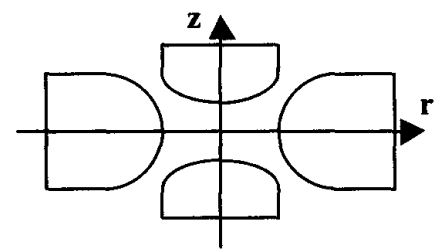

Fig. 2. Electrode axis definitions

The electrode profiles were selected based on [2]. The profiles for the electrodes must be constrained by the following equations in order to maintain the equipotentials of the hyperbolic geometry. The geometry of the vertical electrodes is given by (3) and the inner curve of the ring electrode is given by (4).

$$
\begin{aligned}
& z=\left(\frac{r^{2}}{2}+z_{o}^{2}\right)^{1 / 2} \\
& z^{2}=\frac{r^{2}}{2}-z_{o}^{2}
\end{aligned}
$$

Construction of the EDC is illustrated schematically in Fig. 3.
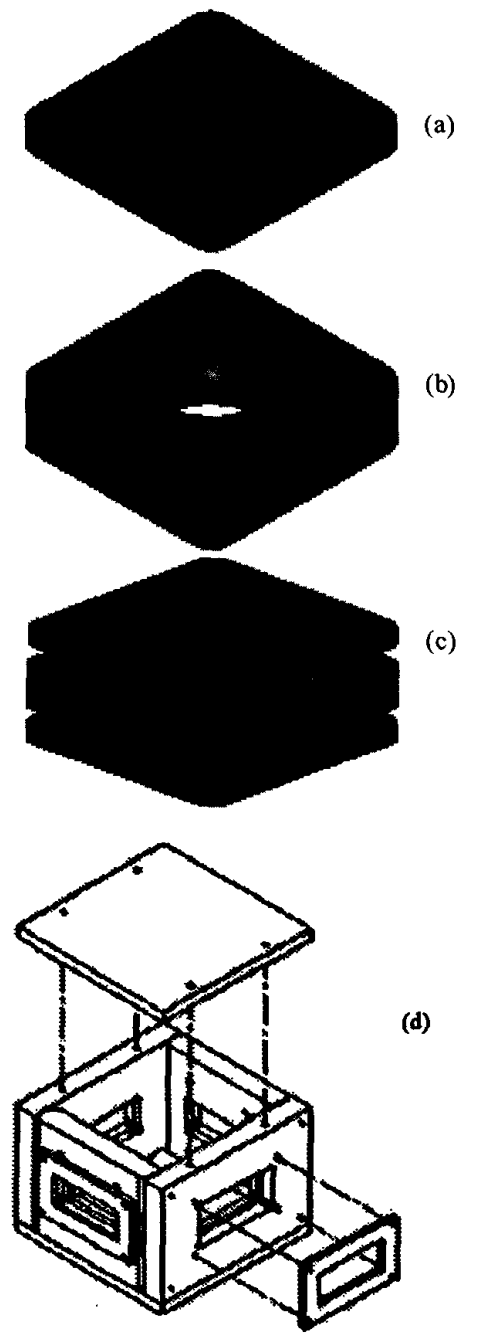

Fig. 3. Chamber assembly. Two end electrodes (a) sandwich a ring electrode (b) to form a completed chamber (c). The EDC is placed in an airtight housing (d). 


\section{B. End Cap Electrode Field Simulations}

Matlab simulations of potential distributions between the vertical electrodes are shown in Fig. 4.

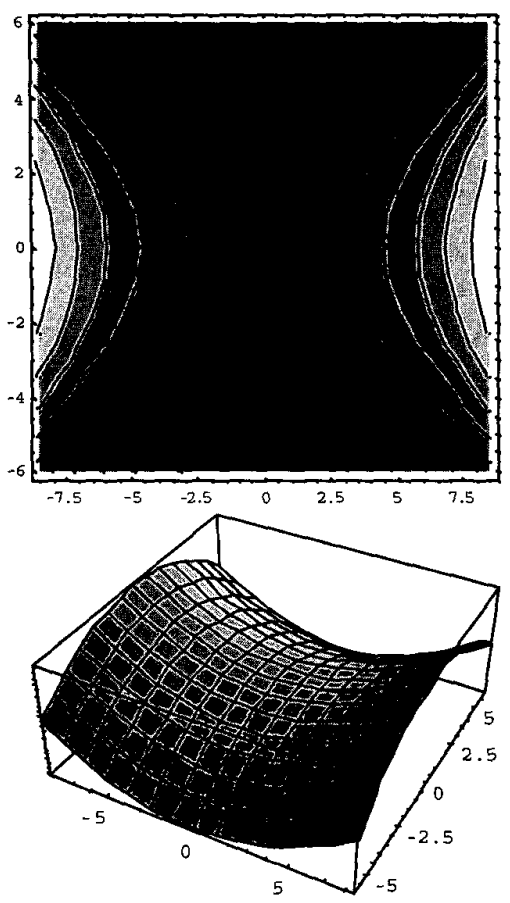

(a)

Fig. 4. Potential contour (a) and 3D (b) plots for simulation results from electrode geometry.

Fig. 5 illustrates the vector E-field and potential difference between the vertical electrodes. For this simulation, the dielectric constant is $\varepsilon_{0}$ and the space charge density is zero.

\section{Position Control System}

Our control approach adopts a hybrid strategy developed from [1] and [4]. A He-Ne laser acting on a linear array is used to locate the particle position (see Fig. 1). The control algorithm is implemented in the controller, which drives a variable high-voltage dc amplifier to produce the required potential on the vertical electrodes. The circular ring electrode is driven with a high-voltage excitation to provide a horizontal centering field, as well as contributing to the vertical force. The components of the position control system are described in the following sections.

\section{1) Imaging System}

The components in Fig. 1 that make up the imaging system are the $\mathrm{HeNe}$ laser, the particle, a diverging lens, the linear array, the linear array driver, and the position sensor. Fig. 6 illustrates the imaging system as a generalized instrumentation system, viewing the $\mathrm{HeNe}$ laser, the particle and the lens as the "physical system", and the linear array and its accompanying driver as a transducer.

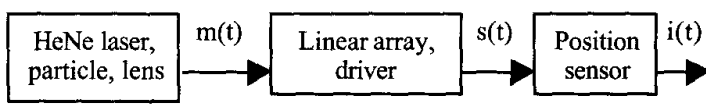

Fig.6. Generalized block diagram of the imaging system.

The imaging system shown in Fig. 6 serves to translate the measured signal (particle location) into a signal that drives the position control system. A HeNe laser is directed towards the linear array. If the particle is in the path of the laser, it will cast a shadow on a diverging lens that distributes the image along the length of a linear

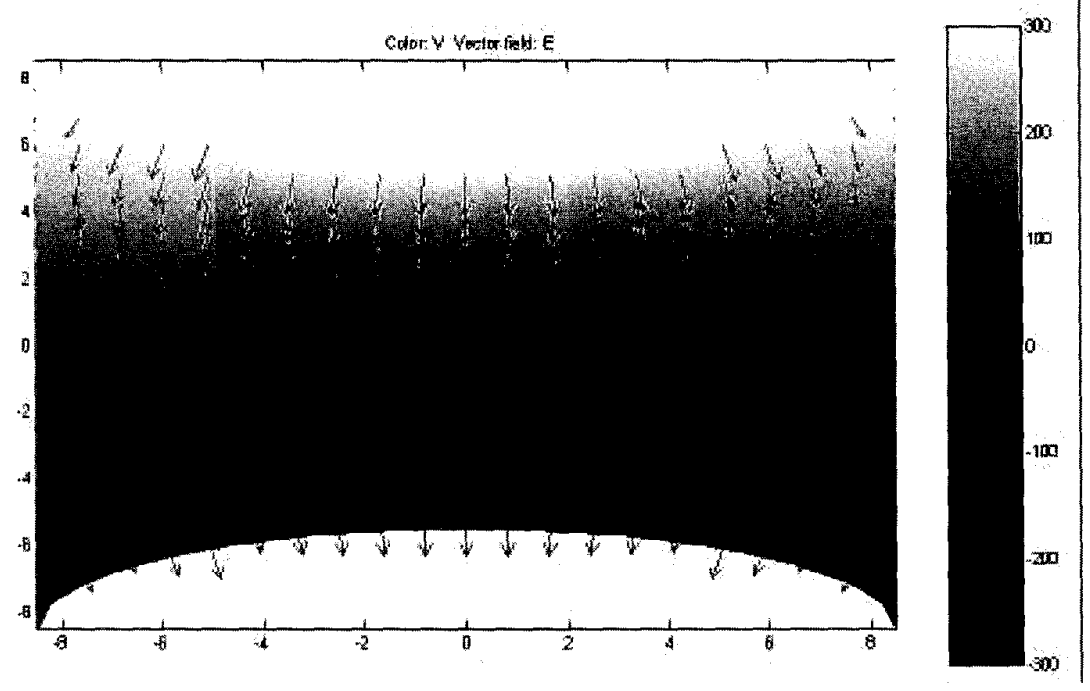

Fig. 5. Electric field simulation for a cylindrical geometry as defined in (3). 
array. The shadow of the particle is the time-varying measurand signal, $m(t)$. Conversion of the measurand is performed by the linear array, with the help of its associated driver to produce $s(t)$. The position sensor instrument then acquires this electrical signal and transforms $s(t)$ to $i(t)$ which indicates the particle position.

The Reticon P Series linear array selected for this project has 2048 elements, providing a $28.728 \mathrm{~mm}$ sensing area [5]. This sensor provides linescan rates up to $135 \mathrm{kHz}$ and data rates up to $40 \mathrm{MHz}$. The spectral response of the P Series is between 200 and $1000 \mathrm{~nm}$. This response supports the spectrum of the HeNe beam $(632.8 \mathrm{~nm})$.

The P Series requires the following six control signals: $\phi_{\mathrm{H} 1}$ (phase 1), $\phi_{\mathrm{H} 2}$ (phase 2), $\phi_{\mathrm{RG}}$ (reset gate), $\phi_{\mathrm{TG}}$ (vertical transfer gate), $\phi_{P G}$ (pixel storage gate), and $\phi_{A B}$ (antiblooming / exposure control gate). A state machine must be designed to provide the timing signals in order to drive the imaging array. The state machine cycles between two major states of operation: integration (acquire image) and line transfer (data exits CCD shift register).

\section{2) Vertical Electrode Voltage Controller}

The purpose of the controller is to regulate the dc voltage potential between the vertical electrodes. The required compensation voltage is a function of the particle properties, particle position and velocity, and the surrounding environment. Using the equations described below and in [1], simulations for the particle movement have been performed in order to evaluate control algorithms that will later be implemented in hardware. The de voltage

$$
V_{D C}=V_{D C}^{0}+\delta V_{D C}
$$

is the summation of the dc voltage required to levitate the particle at the center of the chamber and an offset voltage term.

When identifying the relationships between the system variables, the system can be modeled by a finite number of coupled, first-order differential equations (state equations)

$$
\begin{aligned}
& \not \notin=f_{1}\left(t, x_{1}, \ldots, x_{n}, u_{1}, \ldots, u_{p}\right) \\
& \not \&=f_{2}\left(t, x_{1}, \ldots, x_{n}, u_{1}, \ldots, u_{p}\right) \\
& \mathrm{M} \\
& \not \underset{n}{\&}=f_{n}\left(t, x_{1}, \ldots, x_{n}, u_{1}, \ldots, u_{p}\right)
\end{aligned}
$$

where $\alpha_{i}$ is the derivative of the state variable $x_{i}$ with respect to time $t$, and $u_{l} \ldots u_{p}$ are the inputs to the system
[6]. The state variables $x_{1} \ldots x_{n}$ are key variables that serve to completely descibe the dynamic behavior of the system. The state variables determine the future behavior of the system when the present state of the system and the excitation signals are known [7].

An important concept in dealing with the state equation is the concept of an equilibrium point. A point $x$ $=x^{*}$ in the state space is said to be an equilibrium point of the unforced stated equation (defined as the system with all of its inputs set to zero)

$$
\not k=f(t, x)
$$

if it has the property that whenever the state of the system starts at $x *$ it will remain at $x^{*}$ for all future time.

The process of evaluating potential control algorithms begins with the equations of the motion of a charged particle in the EDC for the vertical and horizontal directions, shown below respectively [1].

$$
\begin{aligned}
& \frac{d^{2} \xi}{d \tau^{2}}+2 k \frac{d \xi}{d \tau}-2 E \xi \cos (2 \tau)-h=0 \\
& \frac{d^{2} R}{d \tau^{2}}+2 k \frac{d R}{d \tau}+E R \cos (2 \tau)=0
\end{aligned}
$$

$h$ and $E$ are electrodynamic field strength parameters, and $k$ is a drag force parameter.

The experimental state variable $I_{D C}^{\&}$ is derived for each control algorithm. The state equations that describe the motion of the particle are defined in (10).

$$
\begin{aligned}
& x_{1}=\xi \\
& x_{2}=\xi \\
& x_{3}=R \\
& x_{4}=R^{k} \\
& \not \&=x_{2} \\
& \not \&=q_{1} \\
& x=x_{4} \\
& x=\frac{9}{3}
\end{aligned}
$$

The vertical direction and velocity of the particle are defined above as the state variables $x_{1}$ and $x_{2}$. The similar horizontal variables are $x_{3}$ and $x_{4}$. The remaining terms are derivatives of the state functions. The final step is to evaluate the response of the system by manipulating various control algorithms. 

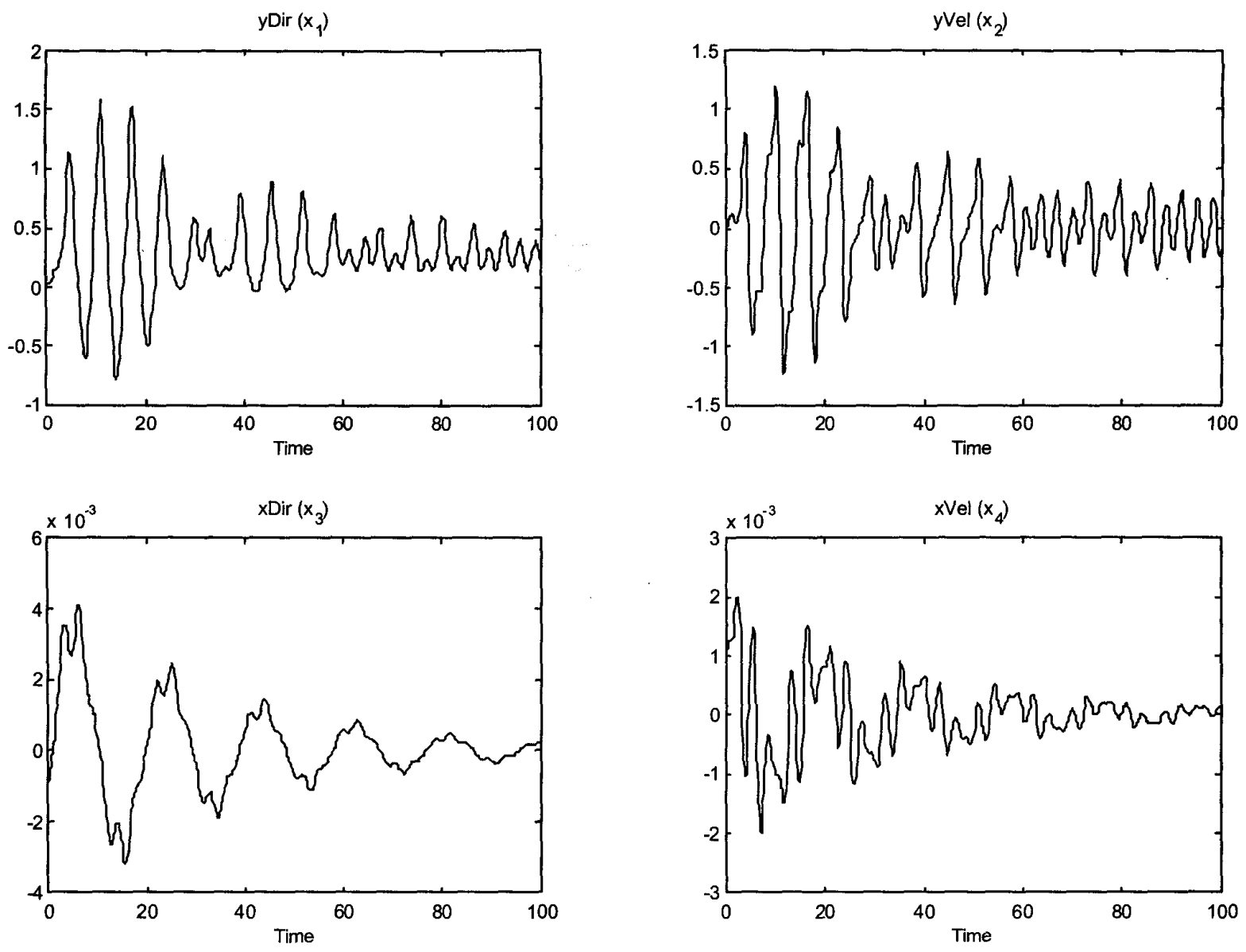

Fig. 7. Control algorithm simulation.

The expression that defines the controller algorithm is

$$
C(s)=\frac{V_{D C}(s)}{\xi(s)} .
$$

The validity of suggested control models was investigated using MATLAB. The following describes an example simulation of a lead controller given by (12), where $K_{c}$ is the controller constant, and $z$ and $p$ are the zeros and poles of the controller function, respectively.

$$
I_{D C}^{\&}=K_{c}\left(\&+z x_{1}\right)-p V_{D C}
$$

The assumptions made for this simulation is that there is no combustion stimulus, the gas inside the chamber is air, and the chamber constant, $c=0.8771$ [1]. It should be noted that the chamber constant must be determined experimentally, as it differs from chamber to chamber. An accurate calculation of $c$ requires measuring the charge of the suspended particle, and evaluating (1). It is presumed that a uniform test particle will be used, with a diameter of $300 \mu \mathrm{m}$ and a density of $1200 \mathrm{~kg} / \mathrm{m}^{3}$. Also, the ac voltage is initially fixed to 2000 volts $\mathrm{rms}$ for most of the simulations. The time span for each trial is initially set to 100 intervals, and can be adjusted as desired.

A starting point in analyzing the simulations is to observe the simulation when the system is operating around some target values. It is initially assumed that the particle will favorably react when the ranges for $E$ and $k$ are $0.1-2$ and $0.2-3$, respectively [1]. By varying the many remaining variables (e.g. initial dc voltage, initial vertical and horizontal positions and velocities of the particle, ac field potential and frequency, time intervals, zeros, poles and controller gain), the control algorithm can be examined for performance characteristics. Fig. 7 shows an example simulation of a control algorithm, depicting the vertical and horizontal positions and velocities. Some of the parameters set for this simulation are as follows: an initial dc potential of 300 volts, the particle is $6 \mathrm{~mm}$ from the null point, the ring electrode is 2060 volts rms at $38 \mathrm{~Hz}$, and the controller parameters are $p=10, z=0.1$ and $K_{c}=5$. 


\section{FUTURE WORK}

This paper described the preliminary design of an electrodynamic chamber. An EDC provides a means of creating an electrodynamic field to levitate charged particles. By isolating a single particle in a chamber, many properties of the particle can be studied. An optimal control algorithm must be determined through more simulations, fabrication of the electrodes is to be completed, and thorough testing of the EDC will be done before it is used for particle analysis. Phase 2 design work will involve the development of prototype elements with testing. Although there is still much work for this project to reach completion, the EDC will prove to be a valuable tool for combustion research.

\section{ACKNOWLEDGMENT}

C.B. thanks our contacts at Department of Energy/Federal Energy Technology Center (DOE/FETC), Dr. Dan Maloney and Dr. Essi Monazam. Dr. Maloney is the main investigator who built/developed the experiment, and Dr. Monazam is the person who is currently most involved with running the EDC system.

\section{REFERENCES}

[1] E. Bar-Ziv and Y. Weiss, "Further development of the electrodynamic chamber for studying singleparticle oxidation and non-uniform shrinkage of char particles," Combustion and Flame, 95:362-73, 1993.

[2] E. Bar-Ziv, A. Sarofin, "The Electrodynamic Chamber: A tool for Studying High Temperature Kinetics Involving Liquid and Solid Particles," Prog. Energy Combust. Sci. 17: 1-65, 1991.

[3] E. Bar-Ziv, R. Spjut, A. Sarofim, and J. Longwell, "Electrodynamic thermogravimetric analyzer," Rev. Sci. Instrum, 57(8), 1604-10, Aug. 1986.

[4] D.J. Maloney, "An automated imaging and control system for the continuous determination of size and relative mass of single compositionally dynamic droplets," Rev. Sci. Instrum., 60(3): 450-5, March 1989.

[5] Reticon P-Series Datasheet url: http://www.perkinelmer.com/Opto109/downloads/pseriesrl.pdf

[6] Khalil, H. Nonlinear Systems, $2^{\text {nd }}$ ed. Prentice-Hall, Upper Saddle River, NJ, 1996.

[7] Dorf, R., Bishop, R. Modern Control Systems, $8^{\text {th }}$ ed. Addison Wesley Longman, Inc., Menlo Park, CA, 1998. 